\title{
Building a new University at the Intersection of Liberal Arts and Engineering
}

\section{Dr. Sebastian Dziallas, Fulbright University Vietnam}

Sebastian Dziallas is a Founding Faculty Member at Fulbright University Vietnam in Ho Chi Minh City. He completed his PhD work in the Computing Education Research Group at the University of Kent. His research examines characteristics of computer science graduates and uses a highly qualitative, narrative methodology to explore the sense graduates make of their own educational experiences within their wider learning trajectories. Before joining the University of Kent, he graduated from Olin College of Engineering in the United States.

\section{Prof. Naoko Ellis P.Eng., The University of British Columbia}

Naoko Ellis is a Professor in the Department of Chemical and Biological Engineering at the University of British Columbia (UBC). She holds a Ph.D. (UBC, 2003); M.E.Sc. (Western, 1993); and a B.Sc. (Honours, Waterloo, 1991). She is a member of the Association of Professional Engineers and Geoscientists of BC. Her expertise lies in the area of multiphase reaction engineering with emphasis on biomass utilization. Some current projects include: biomass gasification and pyrolysis; $\mathrm{CO} 2$ capture, including chemical looping combustion; pyrolysis product utilization; and biofuels. She is also interested in sustainability education and transdisciplinary learning.

\section{Dr. David Robert Bruce P.E., Fulbright University Vietnam}

Dr. Bruce has a passion for technology development with a focus on empowering society through altering perception and perspective. He holds a B.A.Sc. in Environmental Engineering from the University of Waterloo, an M.A.Sc. in Materials Science \& Engineering from McMaster University, and a Ph.D. in Chemical and Biological Engineering from the University of British Columbia. This varied engineering education has taught him to view our world through a multifaceted lens. Dr. Bruce has studied and worked in the alternative energy field for 15 years in a variety of disciplines, with industrial experience in both large tech companies and start-ups. Dr. Bruce is keen to share his understanding into underlying physical science and how to use it to actualize engineering and bring innovation from conception to production. Dr. Bruce is an avid sculptor, painter, and photographer and he hopes to pioneer integration of fine arts with engineering at Fulbright to help examine what serendipitous discoveries can be found through these combined fields. 


\title{
Building a new University at the Intersection of Liberal Arts and Engineering
}

\begin{abstract}
Educating holistic engineers has become the challenge of the $21^{\text {st }}$ century as society grapples with increasingly complex problems. An opportunity has emerged in building brand new liberal arts, science, and engineering programs at Fulbright University Vietnam, a new institution in Vietnam. Founding faculty members have engaged in a "co-design year" to prototype and iterate all aspects of this new university together with students and staff. In this paper, we reflect on the co-design year and present the main considerations that have driven the design of the undergraduate program.
\end{abstract}

\section{Introduction}

There has been increasing awareness to "re-engineer" engineering education as society grapples with increasingly complex, ill-structured, and adaptive problems, such as water scarcity, global pandemics, climate change, poverty, and the loss of biodiversity, which technology alone cannot solve. These efforts have aimed to provide a holistic engineering education [1], producing Tshaped engineers [2] who possess broad knowledge across disciplines with deep expertise in their domain. We have seen many pedagogical advances, such as team-based learning, problembased learning, experiential learning, and creative learning using virtual reality, to name a few. The core driver for this change stems from the need for engineering education to prepare engineers to stay relevant and to contribute to society in the face of rapid global change and advancement in information and technology.

These trends have been the main motivator for integrating liberal studies and engineering. Bucciarelli and Drew laid out a "design plan" for liberal studies in engineering emphasizing the importance of the social and political context in engineering practice [3]. The key dimensions of learning they identified include: analytical thinking, multiple framing, critical reflection, practical reasoning, as well as engineering dimensions of instrumental rationality, empirical testing, transformation of ideas into products and systems via design [3]. Klein, on the other hand, approaches from understanding the limits of engineering, and draws on engineering students to realize the limits of their knowledge and to learn to engage with others [4]. Working on grand challenges in a global context or complex problems in a local setting requires the command of strong technical skills, as well as key interdisciplinary skillsets, such as problemsolving, team-work, the ability to think in systems, empathy, and being adaptive and openminded [3], [5]. These skillsets are not only complementary to an engineering education [6], [7], but are critical to graduates' adaptability on the job market.

In this paper, we present a case study of Fulbright University Vietnam, a new, private, non-profit university in Ho Chi Minh City. Fulbright is inspired by American liberal arts traditions and offers a liberal arts, sciences, and engineering education with Bachelor degrees awarded after four years of study. It aims to prepare the next generation of engineers to work on global grand challenges. Indeed, the goal of its undergraduate program is "to provide an outstanding educational experience that leaves graduates [...] prepared to thrive as the next generation of 
thinkers, leaders, entrepreneurs, scientists, intellectuals, and artists, prepared for the complex challenges of the 21st century, in service to Vietnam and the world." [8]

At the beginning of this journey of building the university, very little was documented. This is not uncommon for projects such as this, where "building the airplane while flying it" often takes precedence over reporting on what happened. However, we believe that there is value in reflecting on and making sense of the process, as well as in documenting the lessons learned.

In the following, we then focus on the main considerations that have driven the design of the undergraduate programs: a co-design process that involved students in the design of culture and curriculum at the institution; the use of user-centered design and scrum methodologies; and the inclusion of a liberal arts core experience for all engineering students.

\section{Context}

As an entirely new university, Fulbright is situated among a number of similar efforts to create new models of education, such as Olin College of Engineering in the United States, the New Model in Technology and Engineering (NMiTE) in the United Kingdom, and Quest University in Canada. However, unlike Olin and NMiTE, it reaches beyond a single disciplinary focus on engineering and incorporates engineering into a liberal arts environment similar to Smith College, Swarthmore College, and Cornell University.

The university's roots can be traced back to the Fulbright Economics Teaching Program (FETP), which was established in 1995 in partnership with the Harvard Kennedy School and is now known as the Fulbright School of Public Policy and Management (FSPPM). The school offers master's degrees in public policy, as well as executive education in leadership and management. Building on this program, Fulbright University Vietnam was then formally established in 2016, adding the undergraduate liberal education programs.

The development of the academic program was initiated through a design process, as the resulting program was not intended to be a copy of any one institution or a combination of several existing curricula. In the 2018-19 academic year, Fulbright then conducted a co-design year, which was structured to provide a unique period of time to prepare for the launch of the academic year of 2019-20, when it officially opened its doors to its first graduating class of 113 students.

There are currently 15 faculty members with backgrounds across the disciplines, for a studentfaculty ratio of roughly 7.5:1. In the next few years going forward, the university aims to recruit an additional 130 students each year, with plans to grow the faculty as well, while a permanent campus is being built. It ultimately aspires to reach a steady state of roughly 3000 students in total after 10 years.

The students come from a variety of backgrounds. Many students have recently graduated high school; some even turned down scholarships to study in the United States [9]; but there are also 
others who previously attended other institutions (whether at home or abroad) and decided to join Fulbright instead.

While currently all students in the undergraduate program are domestic students, Fulbright has aspirations to attract international students in the future. The founding faculty team represents a wide range of academic disciplines, from anthropology to computer science, economics, engineering, film studies, history, natural science, and psychology, among others.

At Fulbright, there are no academic departments, and faculty from different disciplines jointly develop courses across the curriculum, including a set of core courses, emphasizing interdisciplinary learning. The institution offers degrees in several areas of study, including the arts, humanities, social and natural sciences, as well as computing, mathematics, and engineering. Fulbright offers a single general engineering degree with several concentrations, for which it is seeking ABET accreditation. This presents a unique opportunity for the integration of the liberal arts and engineering in a truly interdisciplinary manner.

In the following, readers will learn about the authors' experiences developing a new program at the intersection of liberal arts and engineering. With a strong vision to educate the next generation of engineers, we are still learning and growing to understand where the center of gravity may lie for us for a liberal education in engineering.

\section{The Case of Fulbright}

\section{The Co-Design Year}

Fulbright recently completed its co-design year, which saw a group of 54 students (co-designers) working together with faculty members from different disciplines to design and develop both the culture and curriculum of the institution before the launch of the undergraduate program in the fall of 2019. Admission to the co-design year was competitive, with an acceptance rate of $14 \%$. Students ultimately received a half-year of elective credit for their participation in the year.

The co-design approach was inspired by the partner year at Olin College, which was an early collaborator in the establishment of the institution. However, while the co-design year was a deliberate choice to lay the groundwork for the university, at Olin it was more of an accident: The college had already recruited students to join its first graduating class in 2001 when it discovered that the buildings on its campus would not be ready in time [10]. Yet, the experiences during the partner year proved essential in shaping the college. As Olin's president wrote in a recent article: "As a result of the early experiments [during the partner year], the curriculum began to decouple content knowledge and technical courses from the dominant definition of engineering and consider engineering as a way of thinking, not a body of knowledge. Equally critical, the Partner Year established the importance of the student voice as a fundamental pillar of the Olin educational program." [10]

In recent years, co-design approaches have become more common. NMiTE, a new institution of higher education in the UK with a focus on engineering and the first new university in the 
country in more than 40 years, adopted a similar approach. At NMiTE during the 2018-19 academic year, participants were part of a "Design Cohort," which "comprised a group of school-leavers and graduates and helped to co-design and co-create NMITE's way of learning and shaping the learner experience. These 25 enterprising individuals came from across the UK and international locations to take part in this unique opportunity." [11] However, unlike at Fulbright, the design year at NMiTE was structured in such a way that participants remained at the institution for shorter periods of time.

The primary goals of the co-design year for Fulbright were to:

- develop and test prototypes of courses;

- develop key features of the curriculum and program, especially those required for launch;

- develop active and engaging classes;

- establish a culture of ownership and engagement across the university, particularly for students.

The co-design year was divided into several modules (Figure 1). While the modules in the first half of the year were generally pre-planned, there was room left in the second half of the year to adjust the schedule and to respond to any needs that emerged over the course of the first modules.

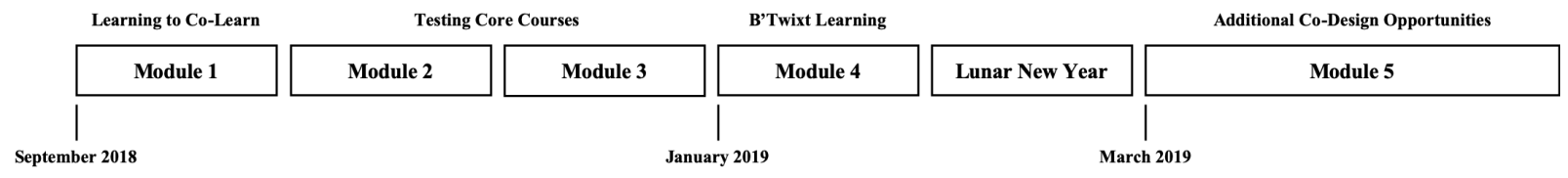

Figure 1. Schedule during the co-design year.

Module 1: Learning to Co-Learn

The first module in the co-design year involved a course titled Learning to Co-Learn. In the time leading up to the start of the co-design year, faculty felt that it was necessary to develop an extended onboarding experience to introduce students to the year ahead. This course experience was co-taught, with two to three faculty from different disciplines and backgrounds working together with a group of around ten co-designers and had the following learning objectives.

Students should:

A. Uncover and articulate their motivation to be part of the co-design year.

B. Develop and articulate their expectations for themselves and for each other, as well as faculty.

C. Develop skills to provide effective feedback (e.g., active listening, etc.).

D. Be able to identify strengths and weaknesses in lesson plans to facilitate reflection on the learning process and future course development.

E. Develop a trusting community between and within faculty and students.

F. Be able to articulate and develop skills to be effective co-learners (e.g., reflection, aspiration, self-awareness, curiosity, vulnerability, growth-mindset). 
G. Participate in a sprint to design a lesson plan with relevant pedagogy.

As part of the course, students discussed different definitions of integrity and took part in activities to "pull back the curtain" - that is, they learned about the pedagogical choices faculty made for a particular lesson, which included reviewing lesson plans, and also ultimately designed their own lessons.

\section{Modules 2 \& 3: Testing Core Courses}

The second and third modules, which each lasted four weeks with a week-long break in between, provided opportunities to test existing core course prototypes that had been developed over the summer. Fulbright was still experimenting with a block schedule at the time, which was originally pioneered by Colorado College [12], [13]. As part of this system, co-designers were participating in a single class for four weeks, with three hours of classroom time on four out of five days per week. ${ }^{1}$ (The one day on which there were no scheduled classes was Thinking Wednesday, which was intended to make room for students to engage in extracurricular activities.)

Students were randomly assigned to their courses in the second module. As all courses offered were core courses, every student would have to take them eventually during their time at Fulbright. This also meant that faculty were able to elicit different perspectives from students, including from those who would not have opted to participate in a particular course. In the third module, students were then given the opportunity to express a preference.

Many of the courses offered during modules 2 and 3 were co-taught by two faculty members from different disciplines. The courses varied in the extent to which they provided co-design opportunities. On one end of the spectrum was a course called Vietnamese Studies, which ran as a complete course that was tested as a whole. On the other end of the spectrum was a course called Rhetoric, in which students took ownership over the course syllabus and explored, debated, and defined which topics should be covered, while examining their own assumptions, background, and culture [14]. To this day, the experience in the Rhetoric course is often described as a moment of true co-design.

In the engineering realm, a group of students took part in a course called Creating \& Making. In the course, students worked in small groups throughout the term to build toys to teach eight-yearolds about a topic of their choice [15]-[17]. Students learned about the user-centered design process, ideation techniques, modern fabrication technologies (including CAD), and scrum methodology. Importantly, these kinds of project-based experiences are a part of the entire engineering curriculum and are not limited to advanced courses.

\footnotetext{
${ }^{1}$ At this point, the institution has adopted a quarter system, where students take two courses for eight weeks, with each course normally meeting for a total of six hours per week.
} 


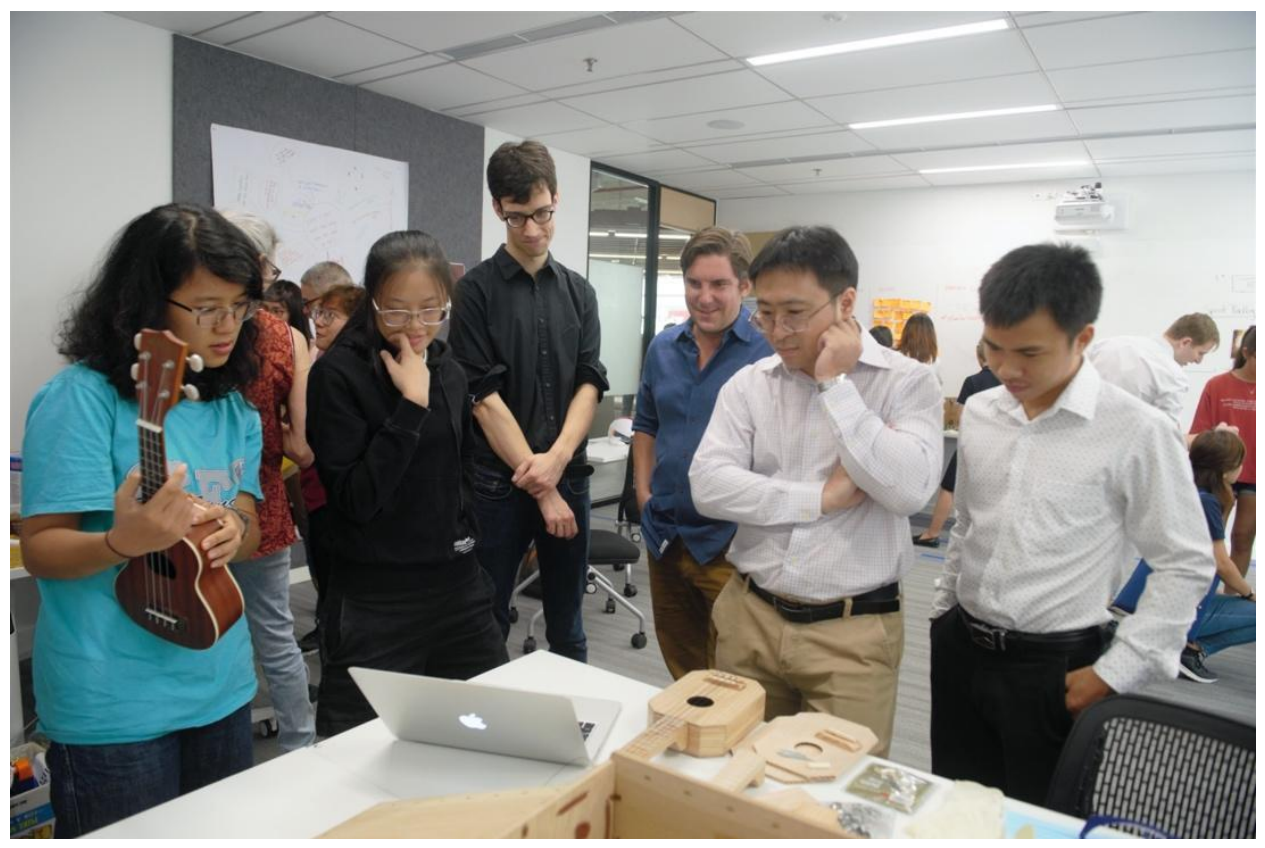

Figure 2. Creating \& Making final project presentations during the co-design year.

This course fell in the middle of the spectrum in terms of students taking ownership of the course content: On the one hand, it was intended to test whether such a project-based course could run given the limited resources available during the co-design year (for instance, a makerspace had not been built and much equipment was yet to be procured) and in order to identify any issues with institutional processes (each team in the course is given a budget for their project, which meant institutional policies had to be established accordingly).

Faculty also used this opportunity to experiment with the inclusion of an art component in the course. Sequentially, the class moved from introduction to art and perception, defining art, identifying design inspiration to introduction to user centered design. Students were assigned readings on aesthetics and perception and subsequently went on a "design inspiration hunt," where they were asked to research, document, and present about a design that was inspired by something. The self-organized student groups managed to present their ideas connecting design and inspiration in creative ways. However, based on student feedback solicited throughout the course, these activities did not appear to substantially support new connections between art and design. Following this experiment and based on the feedback from students, faculty decided to incorporate art into a separate core course, entitled Visual Culture, which was in turn developed in collaboration with students later during the co-design year.

\section{Modules 4 \& 5: B'Twixt Learning \& Beyond}

Following these courses, at the half-way point of the co-design year, in module 4 , students took part in an experience called B'Twixt Learning. As the course description noted: "We decided that this balance point was a good time to stop and do some deep reflection to make the implicit skills you've developed become visible, and to arm you with some professional development skills going forward." In this module, students had the opportunity to investigate their own interests, to 
develop their CVs, and to prototype a Compelling Academic Problem plan (which describes their proposed major courses and a capstone project), as well as ample time for reflection.

Finally, module 5, following spring break, provided space (across several two-week segments) for faculty and students to prototype new courses, to test existing ideas, and to get to know each other in reading groups. Faculty used this time as an opportunity to work with students, for instance, to develop the transition to major experience, to co-design a new arts core course and a revision to the Logic \& Limitations core course, as well as to run several interdisciplinary courses.

\section{Reflections on the Co-Design Year}

The aim of the co-design year was not solely to produce fully developed course syllabi, but also to build the culture of the institution. These goals also emerged in a survey that was conducted at the beginning of the year with all founding faculty members. Specifically, participants were asked about their understanding of the goals of the co-design year. One participant described it as "working together with students to develop the curriculum and culture of the institution," whereas another was looking "to discover our shared identity and shared vision."

Others focused on the need to develop institutional structures, such as policies and procedures. While developing and testing courses was seen as one important element of the co-design year, it was often in the light of student engagement and, particularly, in terms of building a learning community. As one faculty member observed: "This means not just running classes, but actively engaging students to reflect on and to design aspects of the institution with a large amount of autonomy." Another participant similarly noted that they were aiming to "effectively engage students as co-designers, establish a strong Fulbright community, including students, faculty, staff, and friends of the university, effectively adapt a liberal arts education model to the local context, such that Vietnamese culture is intertwined throughout the curriculum."

Both faculty and students then entered the co-design year embracing the unique opportunity to build the undergraduate program by designing the curriculum, cultivating a culture of inquiry, developing a sense of place, and experimenting with innovative pedagogy together. In this, we found the co-design approach to curriculum development, intimately involving students in the design of culture and curriculum at the institution, to be uniquely empowering.

\section{Use of User-Centered Design \& Scrum Methodologies}

Another important aspect of the co-design year was the use of user-centered design and scrum methodologies both in course design and as content students were exposed to.

Over the summer, faculty initially worked in small teams to develop the core courses which were then subsequently tested and revised as part of the co-design year. These teams of faculty used the scrum framework, which is commonly used in software development. It is a "framework for completing complex projects. Scrum originally was formalized for software development projects, but it works well for any complex, innovative scope of work. The Scrum framework is 
deceptively simple." [18] Examples of such complex work include, for instance, product design and policymaking [19]. (Scrum was also used in the development of a prototype of the institution's course catalogue.)

Scrum generally involves a series of week-long sprints, during which team members work on tasks identified during a sprint planning meeting under the facilitation of a scrum master. These tasks are tracked on a so-called scrum board (which reflects all tasks for a sprint, as well as who is working on them and how long their completion is expected to take) and are discussed during a daily stand-up meeting (which team members use to update each other on their progress and plans for the day ahead). At the end of each sprint, there is commonly a read-out, during which team members share their work with the wider community, as well as a retrospective, which is intended as a time to identify lessons for future sprints. (Work by Knapp et al. provides a recent introduction to the concept of sprint [20].)

Throughout each sprint, faculty tested elements of the courses they were developing with students (who had joined the institution for an English language program over the summer) in order to gather feedback.

There are several benefits associated with using scrum in the design of courses. First, it requires that all participants (in this case, faculty) clearly define their goals for the sprint at the beginning of the week. Second, setting aside dedicated time for the sprint allows team members to focus on the project at hand without outside distractions. Third, the transparency afforded by the daily stand-up meetings means that team members are held accountable to their tasks and that potential roadblocks are identified early on, so that they can be resolved effectively.

Finally, scrum produces a minimum viable product in a shorter period of time than is commonly the case in academia. Indeed, for faculty, the ability to create a course prototype in a single week was uniquely empowering. Of course, these courses were not finished after a single week-long sprint. However, the work provided the opportunity for further testing and refinement in subsequent sprints. For instance, the initial sprint on the Creating \& Making core course resulted in a course syllabus, activity and assignment descriptions, as well as a student journey throughout the course. 


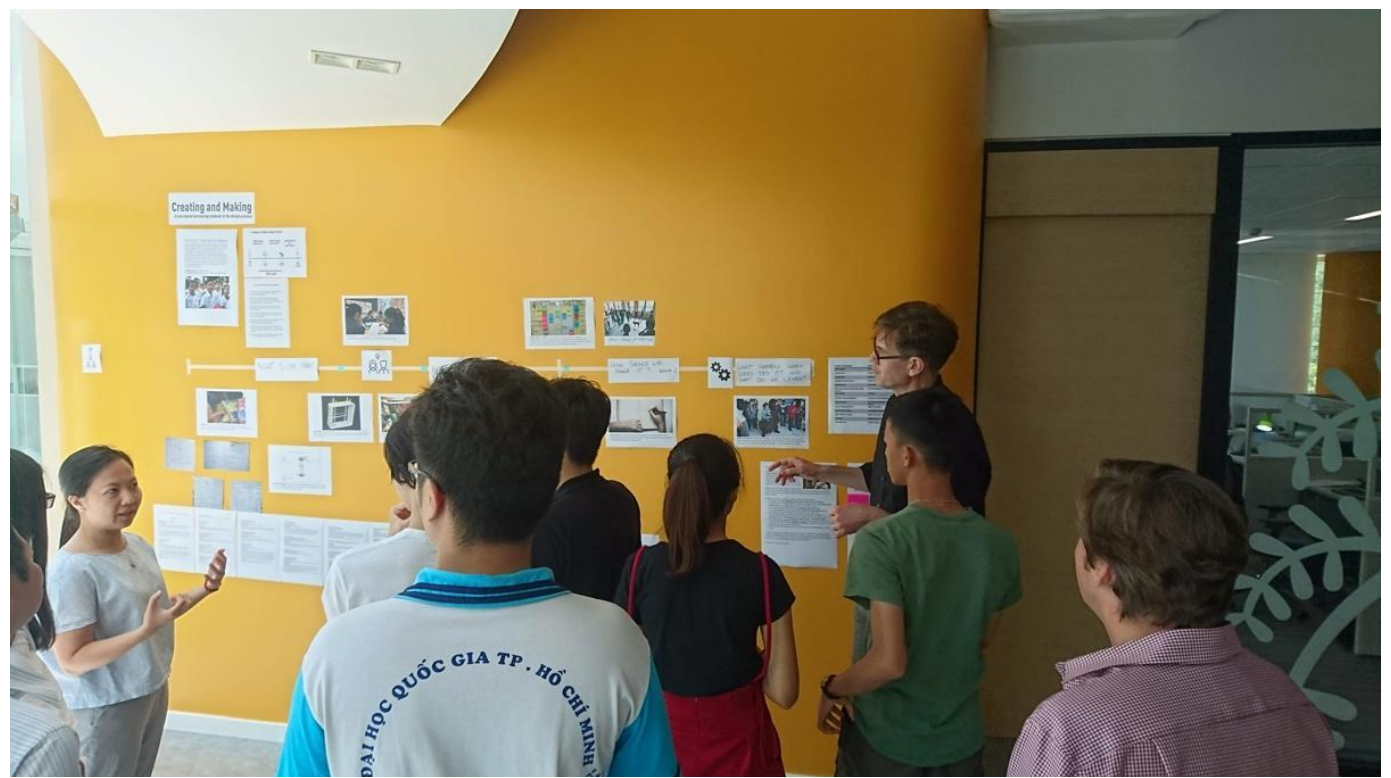

Figure 3. Read-out following the first Creating \& Making sprint during the co-design year.

But the use of scrum was not just limited to faculty work. In the aforementioned Learning to CoLearn course, students were asked to draw on what they had learned about different pedagogical approaches to design their own lessons using scrum. These lessons were designed to teach faculty who had just joined the university about life in Vietnam.

And while Learning to Co-Learn was a one-time experience designed for co-designers, scrum has also found its way into other parts of the curriculum: Today, it is a core part of the Creating $\&$ Making course and, as such, something that all students experience. (Students are asked to complete their projects using scrum methodology.)

Students themselves articulated the effectiveness of this approach in their reflections at the end of the course:

"Moreover, doing scrum becomes my favorite team-working way because of its efficiency and transparency."

"What I like the most about this Creating and Making course is the application of Scrum Model. This model is very effective and helps my whole team keep up with all the tasks done either by themselves or other team members. There is no hard to find an application of this model in any other teamwork, especially in a place like Fulbright where the majority of our big assignments are done in teams. This is also helpful for me moving forward out of Fulbright. I can totally apply this model for myself to update and regularly check-in on my goals and unfinished tasks. Scrum Model is a great tool that students should know to improve their productivity."

"To do the [project], I experimented with a lot of fundamental elements for engineering, especially the Scrum method, the Ideation process, and the Arduino code. I enjoy Scrum 
and also apply it to my personal organization with team members. As well as me, the team members startled when done for the first time but quickly found it effective in teamwork. It is not only to increase ownership in each person and reduce comparison and prejudice with other members. Having a Scrum Master cares about the progress of the work, the participation of each member and the atmosphere of the team to help people become more united."

"[...] we have done successfully in building and developing the scrum framework right in the fresh start of class. It helps not only myself but also my teammates to equally share tasks, optimize flexibility, creativity, and productivity after a sprint per week."

\section{Liberal Arts Core Experience for All Students}

In designing the core courses, interdisciplinary teams of faculty worked alongside co-designers in learning how to create a curriculum for the context of the institution. The resulting seven core courses that all students, regardless of major, are expected to complete by the end of their second year of study, are intended to transcend traditional disciplinary boundaries and to expose students to different ways of thinking. They are:

- Rhetoric

- Scientific Inquiry

- Vietnamese Studies

- Visual Studies

- Ethics and Moral Philosophy

- Logic \& Limitations

- Creating \& Making

This common core experience presents a unique opportunity at Fulbright, where students with different interests share the same set of core courses before they cultivate their disciplinary identities.

This means that students with a particular interest in engineering are exposed to other students' perspectives and different ways of thinking, which they would not have encountered in a traditional engineering program. Conversely, students who previously had not considered engineering as a major may become exposed to and engaged in engineering-based design and teamwork based on their experience with the core courses. The following reflection from Creating \& Making captures the student's appreciation for crossing disciplinary boundaries in tackling problems.

"I am not good at engineering [...]. But our final product is our team's effort, not a single person. Four of us come from different backgrounds: computer science, engineering, art and marketing. We learned how to harmonize that difference. We learned from each other, I learned from every members of my team and we learned together." 
Following their core courses, students select two three-course sequences, the so-called streams, that are designed to open up the major. Following the completion of their stream courses, students should be able to defend their choice of major courses during their Transition to Major. In their third and fourth year of study, students then complete a total of eight major courses, six electives, as well as a capstone project consisting of two courses.

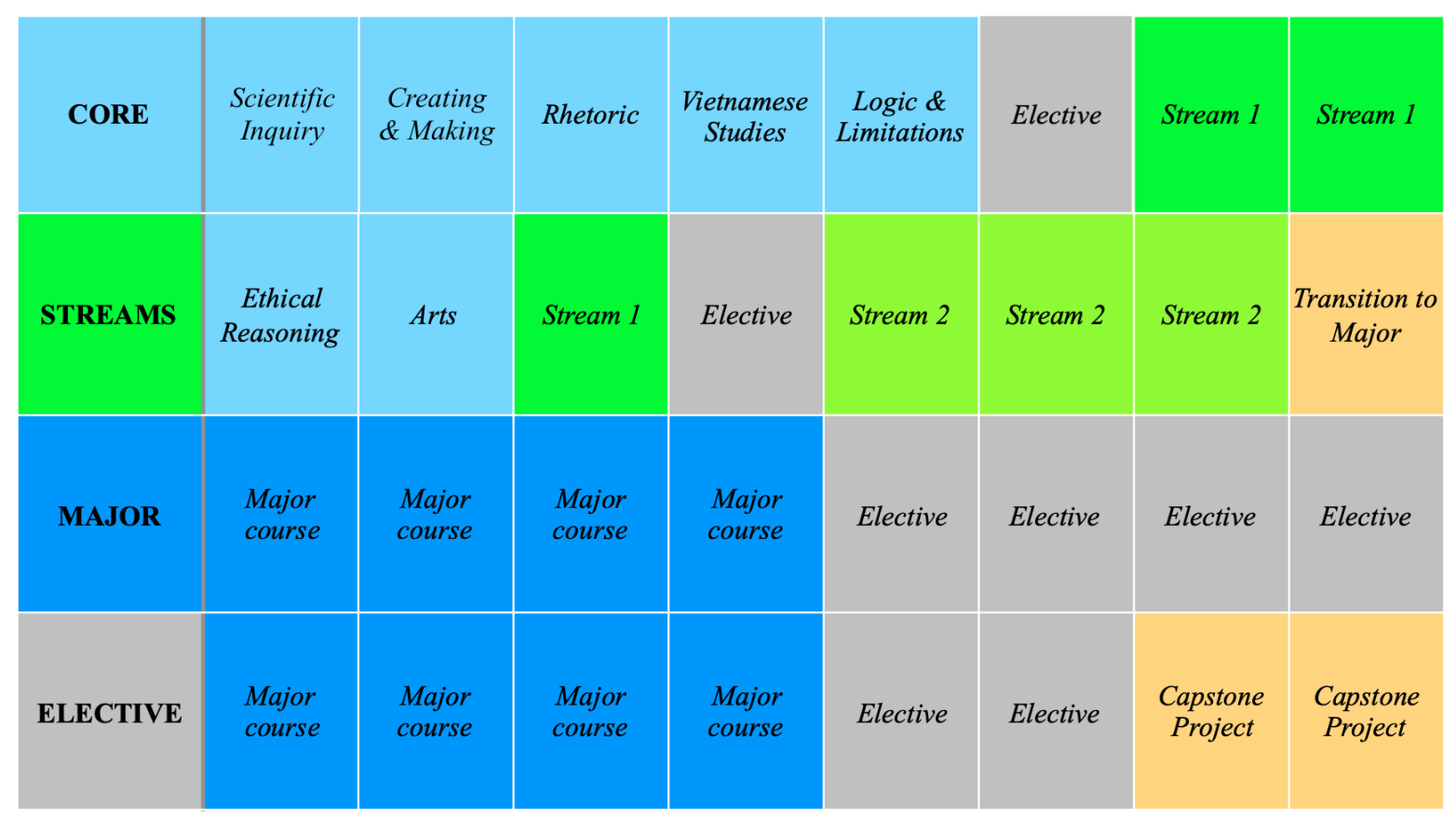

Figure 4. Curriculum map outlining core, stream, major, and elective courses during a four-year degree.

\section{A Word on Assessment}

Fulbright continues to cultivate unique ways of assessing its programs, including the co-design year. One of the main aspects it introduced early on in the co-design year were competencies. Each course would have several associated competencies, which could then be tracked for each student. The competencies are: innovative and creative thinking, collaboration, reasoning, critical thinking, effective communication, civic engagement, lifelong learning, and ethical reasoning. However, this work is still at an early stage. (The university has, for example, experimented with incorporating competency assessments directly into its Learning Management System.)

In terms of evaluations of teaching, rather than using a traditional survey-based student evaluations of teaching, students submit a reflection of no more than 500 words to the following prompt:

Please upload a reflection on the quality of your learning in this course. This reflection should be no more than 500 words. In general, we're interested in hearing how your learning progressed this quarter, what helped you learn, what hindered your learning, 
how much effort you put into the class, what you will do differently in subsequent quarters, and what you need to leave behind. Please be frank. This is graded pass/fail, and designed to provide you with an opportunity to reflect on your learning. When we read these, we use them to reflect on the class from faculty's perspective as well, which makes these a valuable source of data for us.

Faculty are then asked to write a reflection based on the reflections submitted by students. This method of learning assessment contributed to not only reinforcing the culture of reflection, but also cultivated students' awareness of their own learning and their contribution to shaping the perspective of faculty going forward.

Beyond these assessments, Fulbright repeatedly collected additional data during the co-design year, including an English assessment and measures of emotional intelligence and locus of control, among others. Questions of assessment are, of course, also intimately related to the topic of accreditation. From the start, it was decided that Fulbright would seek ABET accreditation for its engineering program. At the intersection of liberal education and engineering education, ABET has been called the "elephant in the room" [21]. While faculty at Fulbright have reviewed different ABET-accredited engineering curricula, including of Olin College, this work is ongoing. However, a detailed discussion of these efforts is outside the scope of this paper with its focus on the co-design year.

\section{Building Faculty Capacity}

The unique opportunity of the co-design year lent itself to attracting faculty who were curious and keen to explore new possibilities in building a university and re-imagining higher education. Indeed, the recruitment of faculty who would be able to work and thrive in an environment that transcends disciplinary boundaries was an important consideration in the recruitment process. Thus, the process differs significantly from traditional hiring processes. During their on-campus interviews, faculty candidates are expected to directly engage with students and to work with other candidates (from other disciplines) to co-design courses which are then presented to students. Candidates are also expected to reflect on their experiences during the interview, encouraging them to be learners themselves.

Once on-board, all faculty went through so-called Instructional Skills Workshops (ISW), which are a "response to the challenge of ensuring university and college teachers have appropriate instructional skills" [22], and participated in discussions on how to co-teach courses with faculty from different disciplines. In designing the curriculum during the co-design year, faculty were challenged to critically examine their own disciplinary assumptions and pushed beyond traditional disciplinary boundaries, promoting them to think laterally and creatively, thus creating an interdisciplinary environment of learning. Furthermore, as part of the co-design process, faculty had to let go of their traditional role as experts in the classroom and instead be open and even feel vulnerable in working with students. The co-design year has given faculty an opportunity to examine their role, beliefs, and values around teaching and learning. 


\section{Challenges and Opportunities}

There are, of course, many challenges and open questions associated with starting a new institution from scratch. At times, it has been difficult to explore - and to pursue — all of the opportunities afforded by the design of a new institution while offering courses, advising, and conducting research at the same time. We also continue to ask ourselves fundamental questions about how to retain the co-design spirit going forward and how much to grow as an institution in the years to come. And we are still examining the role of engineering at the institution and welcome feedback on that: To what extent can the liberal arts and engineering be truly integrated at the university? In some ways, then, this paper is an invitation to continue these conversations with us.

In terms of practices other institutions - whether newly emerging or already established might adopt, one particularly important aspect was an institutional commitment to iteration, with the understanding that any first "product" — whether a newly developed assignment, process, or entire course - would not be perfect and be revised following tests with and feedback from students.

\section{Conclusions}

Starting a new university from the ground up is, of course, a fundamentally different endeavor than implementing a change to an existing degree program. Yet, we believe that there are elements of the story of Fulbright University Vietnam that are relevant to new initiatives in higher education and existing degree programs alike.

In this paper, we have examined the main considerations that have driven the design of the undergraduate program. They are the institution's co-design process, an approach to course design that relies heavily on user-centered design and scrum methodologies more commonly associated with software development, as well as the creation of an interdisciplinary learning environment with a set of core courses that all students are expected to take.

The co-design process is currently particularly under-explored in the literature. Yet, we believe that it played a crucial role in the development of culture and curriculum. For those at other institutions seeking to adopt a similar model, it is important not to reproduce a co-design year with exactly the same elements as at Fulbright (or, indeed, at Olin), but to embody these values of student-centered design, active learning, and student empowerment in their own ways.

\section{References}

[1] D. Grasso and M. Burkins, Eds., Holistic Engineering Education: Beyond Technology. New York: Springer-Verlag, 2010.

[2] K. A. Neeley and B. Steffensen, "The T-Shaped Engineer as an Ideal in Technology Entrepreneurship: Its Origins, History, and Significance for Engineering Education," presented at the 2018 ASEE Annual Conference \& Exposition, Jun. 2018, Accessed: Feb. 02, 
2020. [Online]. Available: https://peer.asee.org/the-t-shaped-engineer-as-an-ideal-intechnology-entrepreneurship-its-origins-history-and-significance-for-engineering-education.

[3] L. L. Bucciarelli and D. E. Drew, "Liberal studies in engineering - a design plan," Eng. Stud., vol. 7, no. 2-3, pp. 103-122, Sep. 2015, doi: 10.1080/19378629.2015.1077253.

[4] J. D. Klein, "Teaching and learning limits in engineering education," Eng. Stud., vol. 7, no. 2-3, pp. 135-137, Sep. 2015, doi: 10.1080/19378629.2015.1062494.

[5] D. D. Gillette, E. Lowham, and M. Haungs, "When the Hurly-Burly's Done, of Battles Lost and Won: How a Hybrid Program of Study Emerged from the Toil and Trouble of Stirring Liberal Arts into an Engineering Cauldron at a Public Polytechnic," Eng. Stud., vol. 6, no. 2, pp. 108-129, May 2014, doi: 10.1080/19378629.2014.944186.

[6] D. Weichert, B. Rauhut, and R. Schmidt, Eds., Educating the Engineer for the 21st Century: Proceedings of the 3rd Workshop on Global Engineering Education. Springer Netherlands, 2001.

[7] N. Spinks, N. Silburn, and D. Birchall, "Educating Engineers for the 21st Century: The Industry View," Henley Management College, Mar. 2006.

[8] R. Derby-Talbot, "Undergraduate Academic Program Report and Update," Fulbright University Vietnam, Jul. 2018.

[9] Ly Minh Tu, "Fulbright is a choice," Fulbright University Vietnam, Oct. 03, 2019. https://fulbright.edu.vn/fulbright-is-a-choice/ (accessed Jan. 30, 2020).

[10] R. K. Miller, "Lessons from the Olin College Experiment," Issues Sci. Technol., vol. 35, no. 2, pp. 73-75, Mar. 2019.

[11] “Design Cohort," NMiTE. https://nmite.ac.uk/about-us/design-cohort/ (accessed Sep. 07, 2019).

[12] P. Heist and M. F. Taylor, The Block Plan: A Preliminary Report on a Ten-Year Evaluation of the Colorado College Block Plan Format for Intensive Study. 1979.

[13] B. Gose, "One-Course-at-a-Time 'Block Plan' Re-examined by College That Adopted It 25 Years Ago," Chron. High. Educ., vol. 41, no. 47, 1995, Accessed: Jan. 30, 2020.

[Online].

[14] Le Thi Nga My, "Step out of our comfort zone," Fulbright University Vietnam, Jan. 22, 2019. https://fulbright.edu.vn/step-out-of-our-comfort-zone/ (accessed Sep. 07, 2019).

[15] Pham Nguyen Dan Tam, "I was wrong. That class was not a plain sailing.," Fulbright University Vietnam, Jan. 03, 2019. https://fulbright.edu.vn/creating-and-making-i-waswrong-it-was-the-most-stormy-class/ (accessed Sep. 07, 2019).

[16] "Creating and Making Class: The journey of Duno," Fulbright University Vietnam, Apr. 25, 2019. https://fulbright.edu.vn/lop-hoc-ve-sang-tao-va-kien-tao-hanh-trinh-cua-khunglong-duno/ (accessed Sep. 07, 2019).

[17] Nguyen Thi Thuy Dung, "The Ukulele story: How to think like an engineer," Fulbright University Vietnam, Feb. 18, 2019. https://fulbright.edu.vn/the-ukulele-story-how-to-thinklike-an-engineer/ (accessed Sep. 07, 2019).

[18] "What is Scrum?," Scrum Training Institute. https://scrumtraininginstitute.com/learnscrum/what-is-scrum/ (accessed Feb. 02, 2020).

[19] J. V. Sutherland, Scrum: the art of doing twice the work in half the time, First Edition. New York: Crown Business, 2014.

[20] J. Knapp, J. Zeratsky, and B. Kowitz, Sprint: How to Solve Big Problems and Test New Ideas in Just Five Days. New York: Simon \& Schuster, 2016. 
[21] D. P. Michelfelder, "Another elephant in the room," Eng. Stud., vol. 7, no. 2-3, pp. 156158, Sep. 2015, doi: 10.1080/19378629.2015.1062496.

[22] J. P. Foxe, M. Frake-Mistak, and C. Popovic, "The instructional skills workshop: A missed opportunity in the UK?," Innov. Educ. Teach. Int., vol. 54, no. 2, pp. 135-142, Mar. 2017, doi: 10.1080/14703297.2016.1257949. 